\title{
Participando da construção de um projeto pedagógico da enfermagem
}

\author{
PARTICIPATING IN THE CONSTRUCTION OF A TEACHING PROJECT FOR NURSING \\ PARTICIPANDO DE LA CONSTRUCCIÓN DE UN PROYECTO PEDAGÓGICO DE ENFERMERÍA
}

\author{
Silvia Helena Zem-Mascarenhas ${ }^{1}$, Maria Isabel Ruiz Beretta $^{2}$
}

\section{RESUMO}

O presente estudo tem por objetivo relatar a experiência vivenciada pelas autoras enquanto participantes do processo de construção do projeto pedagógico do Curso de Enfermagem da Universidade Federal de São Carlos. É feita uma apresentação do curso, e os aspectos relativos à nova proposta são brevemente relatados no que tange ao perfil do profissional enfermeiro e à estrutura das disciplinas distribuídas em módulos. Procura contextualizar a construção coletiva e as dificuldades relacionadas ao processo de reformulação curricular. A proposta de currículo integrado deverá ser implementada no ano de 2005, representando um esforço comum dos docentes do curso na busca da articulação da teoria e prática sem fragmentação. Fica explícito que para o sucesso da implementação do novo projeto pedagógico serão necessários esforços por parte dos docentes, alunos e estrutura administrativa da universidade, visando a encontrar caminhos que apontem a superação das dificuldades.

\section{DESCRITORES}

Currículo.

Educação superior.

Educação em enfermagem.

\section{ABSTRACT}

The aim of the present paper is to report the authors' experience in the construction process of the teaching project for the Nursing Course at the Federal University of São Carlos. The study presents the course and the aspects related to the new proposal concerning the professional nurse profile, and the structure of the subjects distributed in modules are briefly reported. The collective construction and the difficulties regarding the curriculum reformulation process are placed in context. The integrated curriculum proposal should become effective in 2005 , which will bring about the efforts of teachers in their search for the articulation of theory and practice without fragmentation. In order to succeed, the new teaching project will require efforts from teachers, students and the university administrative staff so that paths to overcome upcoming difficulties can be found.

\section{KEY WORDS}

Curriculum.

Education, higher.

Education, nursing.

\section{RESUMEN}

En el presente estudio el objetivo fue relatar la experiencia vivenciada por las autoras como participantes del proceso de construcción del proyecto pedagógico del Pregrado de Enfermería de la Universidad Federal de San Carlos. Se realiza una presenta-ción del Pregrado y, los aspectos relativos a la nueva propuesta, son brevemente relatados en lo que atañe al perfil del profesional enfermero y a la estructura de las disciplinas distribuidas en módulos. Procura contextualizar la construcción colectiva y las dificultades relacionadas al proceso de reformulación curricular. La propuesta de currículum integrado deberá ser implementada en el año 2005, representando un esfuerzo común de los docentes del Pregrado en la búsqueda de la articulación de la teoría y la práctica sin fragmentación. Queda explícito que para el suceso de la implementación del nuevo proyecto pedagógico serán necesarios esfuerzos por parte de los docentes, alumnos y estructura administrativa de la universidad, visando encontrar caminos que apunten la superación de las dificultades.

\section{DESCRIPTORES}

Curriculum.

Educación superior.

Educación en enfermería.
1 Doutora em Enfermagem. Professora do Departamento de Enfermagem da Universidade Federal de São Carlos (UFSCar). silviazem@power.ufscar.br 2 Doutora em Educação. Professora do Departamento de Enfermagem da UFSCar.

dmirb@power.ufscar.br 
O Curso de Enfermagem da Universidade Federal de São Carlos (UFSCar) foi criado em 18 de novembro de 1976 e implantado através da $64^{\mathrm{a}}$ reunião do Conselho de Curadores da UFSCar, em 17/12/1976. Foi reconhecido pelo MEC/CFE através da Portaria nº 237, de 21/03/1980 e na estrutura da UFSCar está inserido no Centro de Ciências Biológicas e da Saúde, sendo o primeiro curso nessa área. Desde sua criação o curso constituiu-se em Bacharelado em Enfermagem e Licenciatura, sendo essa última de caráter optativo.

O primeiro currículo do curso atendia às exigências da Resolução CFE $n^{\circ}$ 4, de 25/02/1972 e às recomendações da ABEn (Associação Brasileira de Enfermagem) que objetivavam a qualidade da assistência em enfermagem à população brasileira.

Em 1982 docentes e alunos iniciaram um processo de análise do curso como um todo, culminando em uma proposta de mudanças no currículo a qual não chegou a ser implantada.

Após esse período vários estudos e discussões entre docentes do curso e alunos ocorreram, resultando em uma proposta do currículo a qual foi aprovada em 1986, tendo sua implementação concretizada em 1988.

Na nova proposta havia uma preocupação com as características da população e os locais de atuação do profissional enfermeiro, o que a diferenciava da proposta de 1977 cujo enfoque estava nas disciplinas/atividades.

Nesse período (1996-98) o curso passou também por uma avaliação interna e externa, seguindo a proposta do Programa de Avaliação Institucional das Universidades Brasileiras (PAIUB).

Naquela época havia, por parte dos docentes do curso, uma insatisfação com relação ao currículo vigente e, diante disso, iniciou-se um processo de reformulação curricular que foi sendo trabalhado ao longo dos últimos anos.

Ao assumirmos a responsabilidade do cargo da coordenação, no ano de 2002, tínhamos consciência de que o curso precisava concluir este processo. Entretanto sabíamos que a finalização do projeto pedagógico era um grande desafio que teríamos que enfrentar. Da mesma forma, outras universidades públicas e privadas tinham a tarefa de construir um projeto político pedagógico com a inserção do profissional enfermeiro na realidade político social vigente.
Consideramos portanto, que esse percurso foi e continua sendo desenvolvido pelos cursos que primam por uma educação/formação voltada para as novas exigências do atual cenário nacional e tendências da profissão. Neste contexto, buscamos com este estudo apresentar um relato de nossa experiência enquanto docentes do curso de enfermagem da UFSCar e atuantes na coordenação do mesmo de 2002 a 2004, período no qual essa proposta pedagógica foi finalizada.

\section{O PROJETO PEDAGÓGICO}

\begin{abstract}
O projeto pedagógico representa os anseios de educadores e educandos de um curso, voltados para o contexto e necessidades de elaborar estratégias que fundamentem e orientem ações interdisciplinares, tendo como predomínio o interesse de autonomia profissional para agir e interagir, segundo a realidade e demanda da população. Tem como base teorias pedagógicas que consideram a interação entre o curso e o contexto geral em que se insere e traz em sua concepção um compromisso definido no coletivo(1).
\end{abstract}

Quando se pretende construir um projeto pedagógico com características inovadoras, algumas considerações são necessárias, principalmente no que tange à dificuldade que traz a eminência do novo, levando ao enfrentamento dos distintos entendimentos e interesses que as mudanças provocam.

Tendo como pressuposto que a construção de um projeto pedagógico precisa ter entre outros fatores, uma ação docente integrada, consideramos que o mesmo se apresenta como uma forma de orientar as estratégias a serem utilizadas pelo curso como um todo.

Portanto, essa construção almejava um caráter coletivo e, como geralmente ocorre na maioria dos processos de mudança, muitos desafios surgiram exigindo vários momentos de reflexões com vistas a atingir os objetivos do grupo.

A construção coletiva de um projeto pedagógico constitui-se em uma experiência desafiadora para a enfermagem brasileira ${ }^{(2)}$. Para obter sucesso na implementação de um projeto pedagógico deve haver comprometimento dos indivíduos envolvidos no processo, sendo, portanto importante que sua construção seja realizada de forma participativa, refletindo o consenso do grupo da melhor forma $\operatorname{possível}^{(3)}$.

Além disso, uma proposta de reformulação curricular precisa estar baseada na concepção de 
que o currículo significa a trajetória de ações com vistas à formação do enfermeiro e como isso pode ser possível. Existe sempre o desafio de reconstruir o curso, buscando compatibilizá-lo com as necessidades de um mercado de trabalho cada vez mais competitivo, necessitando de profissionais críticos, reflexivos, competentes, com base científica, capacidade de inovação e poder de ação.

No desenvolvimento do processo de reformulação curricular foram adotados como parâmetros a LDB (Lei de Diretrizes e Bases) da Educação Nacional n. 9394/96, a Lei do Exercício Profissional e as Diretrizes Curriculares Nacionais dos Cursos de Graduação em Enfermagem, aprovado em agosto de 2001, do Conselho Nacional de Educação/Câmara de Educação Superior. Foi utilizado também um documento elaborado pela Pró-Reitoria de Graduação da UFSCar em 2002, como proposta de reforma curricular dos cursos da Universidade, levando-se em consideração o contexto demográfico e epidemiológico nacional e municipal no qual o curso está inserido.

Esse processo foi iniciado em meados de 1998 quando docentes envolvidos no curso e preocupados com os seus aspectos pedagógicos, iniciaram o longo processo de reformulação curricular. Assim, a Coordenação do Curso almejou uma reestruturação que pudesse atender tanto à legislação acima citada, quanto às necessidades apresentadas por docentes e alunos em avaliações feitas anteriormente.

O ponto de partida da reestruturação curricular foi uma primeira reunião realizada com todos os docentes de Departamento de Enfermagem e discentes, na qual foram levantados alguns aspectos relacionados à insatisfação dos docentes com o currículo vigente, como também, se existia por parte do grupo o anseio por mudanças. As discussões revelaram que o grupo estava insatisfeito com o currículo do curso e se propunha a enfrentar o desafio de uma reformulação curricular.

Entretanto, a construção do projeto pedagógico constituiu-se em uma tarefa árdua, pois além das dificuldades de se construir o novo, esbarrou-se em desentendimentos entre docentes e até mesmo em momentos de desinteresse do grupo, uma vez que mudanças geralmente implicam em abrir mão da segurança do que se tem pronto e a incerteza de como inovar e de como reconstruir.

Um novo momento do curso precisa significar um "como fazer" diferente e romper com o estabelecido no processo de formação do enfermeiro, ou seja, como romper com as velhas concepções e implantar novas que modificarão não somente a estru- tura curricular, mas também mobilizarão os docentes a adotarem outra postura.

No decorrer do processo de reformulação foram realizadas inúmeras reuniões e várias oficinas de trabalho com a participação dos docentes e discentes, norteadas pelos parâmetros já citados e por textos de apoio que promoveram o caminhar da construção do projeto pedagógico.

Embora tenha sido constituída uma comissão com representantes docentes e discentes para a reformulação curricular esta possuía autonomia limitada pois, como o trabalho caracterizou-se como coletivo, sempre predominava a decisão do grupo em momentos de reunião com os demais docentes do curso. Tal fato nem sempre significava o andamento do processo, porque às vezes o que era resolvido em uma reunião não espelhava a opinião de todos e, na próxima reunião, o processo era novamente retomado.

Mas esta situação não pode ser considerada como uma característica particular do nosso grupo, pois, outros autores relatam as mesmas dificuldades por nós vivenciadas nessa trajetória ${ }^{(2-7)}$.

Com as novas propostas de inovação no ensino de enfermagem, pode se esperar algumas dificuldades em termos de aceitação de mudanças de comportamentos e atitudes, tanto por parte dos professores como dos alunos, pois o processo de romper com o modelo sedimentado do ensino tradicional tende a ser duradouro ${ }^{(8)}$.

No caso do curso aqui apresentado, pode-se dizer que a primeira etapa do processo de Reformulação Curricular do Curso de Enfermagem da UFSCar foi concluída. A seguir são apresentados alguns aspectos desenvolvidos no que tange à estrutura curricular e ao perfil do profissional enfermeiro.

\section{A ESTRUTURA CURRICULAR}

A estrutura Curricular foi organizada em quatro módulos, distribuídos ao longo de quatro anos de curso, com carga horária de $3315 \mathrm{~h}$ onde o aluno, em período integral, desenvolverá as atividades propostas.

\section{Módulo I: sociedade, saúde e enfermagem}

Objetivos gerais: compreender as práticas de saúde, entre elas a enfermagem como práticas sociais; compreender o processo de construção do conhecimento científico e realizar o diagnóstico de saúde de uma determinada população. 
Sílvia H.Zem-Mascarenhas Maria Isabel Ruiz Beretta
Este módulo compõe as seguintes áreas de conhecimento: ciências sociais, filosofia, educação, saúde coletiva, enfermagem e bioestatística. Núcleos e disciplinas desse módulo:

- Integração I

Núcleo Ciências Humanas

* Filosofia da Ciência

* Introdução à Sociologia Geral

Núcleo Bases Históricas e Éticas e Legais da Enfermagem

* Bases Históricas e Éticas e Legais da Enfermagem Núcleo Saúde Coletiva

* Saúde Coletiva (Epidemiologia, Políticas de Saúde, Saúde e ambiente, Diagnóstico de Saúde da População)

* Bioestatística

* Educação e Saúde

\section{Módulo II: instrumentalização para o processo de cuidar do indivíduo}

Objetivos gerais: avaliar as condições de saúde do indivíduo e da família nas dimensões bio-psicosócio-espiritual; desenvolver o processo de cuidar (cuidar/educar/pesquisar) do indivíduo, utilizando instrumento metodológico para sistematizar o cuidado de enfermagem; conhecer as bases conceituais do gerenciamento e suas implicações na saúde.

Este módulo se constitui das seguintes áreas de conhecimento: morfologia, fisiologia, patologia e enfermagem. Núcleos e disciplinas desse módulo:

- Integração II

Núcleo Morfologia

* Anatomia

* Citologia, Histologia, Embriologia

Núcleo Fisiologia

* Fisiologia

* Bioquímica e Biofísica

* Farmacologia

* Nutrição

* Genética e Evolução

Núcleo Patologia

* Patologia Geral e Aplicada à Enfermagem

* Parasitologia

* Microbiologia

* Imunologia
Núcleo Fundamentação do Processo de Cuidar em Enfermagem

* Relações Humanas em Enfermagem

* Bases Metodológicas da Pesquisa em Saúde

* Processo de Cuidar em Enfermagem

* Semiologia e Semiotécnica

* Procedimentos de Enfermagem

* Sistematização do Cuidado de Enfermagem

* Psicologia do Desenvolvimento

* Bases Teóricas para o Gerenciamento em

Enfermagem

* Atenção à Saúde da Família

Módulo III: processo de cuidar, gerenciar, educar e pesquisar em enfermagem

Objetivos gerais: planejar, desenvolver e avaliar ações de enfermagem de caráter individual e coletivo voltadas para a promoção da saúde, prevenção de agravos, tratamento e reabilitação nas diversas etapas do desenvolvimento humano, nos diferentes níveis de atenção à saúde.

Este módulo compõe as seguintes áreas de conhecimento: saúde da mulher, do idoso, saúde mental, saúde do adulto e saúde do trabalhador. Núcleos e disciplinas desse módulo:

- Integração III

Núcleo Atenção à saúde da mulher

* Atenção à Saúde da Mulher

Núcleo Atenção à saúde do idoso

* Atenção à Saúde do Idoso

Núcleo Atenção à saúde mental

* Atenção à Saúde Mental

Núcleo Atenção à saúde do adulto

* Atenção à Saúde do Adulto

* Nutrição na Saúde do Adulto

Núcleo Atenção à saúde da criança e do adolescente

* Atenção à Saúde da Criança e do Adolescente

* Nutrição na Saúde da Criança e do Adolescente

Núcleo Atenção à saúde do trabalhador

* Atenção à Saúde do Trabalhador

Núcleo Gerenciamento

* Gerenciamento em Enfermagem 


\section{Módulo IV: consolidação do processo de formação profissional em enfermagem}

Objetivos gerais: planejar, desenvolver e avaliar ações cuidativas, educativas, gerenciais e de pesquisa de forma integrada e autônoma, vivenciando o processo de trabalho da enfermagem nos diferentes serviços de saúde; desenvolver um trabalho de conclusão de curso sob orientação docente; desenvolver consciência crítica para o exercício ético-legal da profissão.

As áreas de conhecimento envolvidas neste módulo são todas as áreas referidas nos módulos anteriores.

Neste módulo o aluno terá oportunidade de vivenciar a prática profissional na realização do Estágio Curricular Supervisionado em três áreas de atuação: hospitalar, saúde coletiva e em uma área de interesse do aluno, com oportunidade de discutir e refletir criticamente sobre o exercício de sua profissão. Núcleos e disciplinas desse módulo:

- Integração IV

Núcleo Estágio Curricular Supervisionado (ECS)

* Exercício da Enfermagem

* ECS: Planejamento do Trabalho de Conclusão de Curso

* ECS: Área de Interesse e Trabalho de Conclusão de Curso

* ECS: Área Hospitalar

* ECS: Área de Saúde Coletiva

\section{PERFIL DO PROFISSIONAL ENFERMEIRO DA UFSCar}

O Curso de Enfermagem da UFSCar tem como meta a formação de um profissional generalista, que seja capacitado para atuar na coordenação do processo de trabalho da enfermagem, na qualificação de seus agentes e no desenvolvimento de ações cuidativas, educativas, gerenciais e de pesquisa junto ao indivíduo, à família e à coletividade .

Para o desenvolvimento da profissão o enfermeiro deve levar em consideração tanto o perfil epidemiológico da população, como a legislação e as instituições onde as ações serão realizadas. É importante que tenha um comprometimento ético e político com aspectos relacionados à valorização e defesa da vida, bem como com a preservação do meio ambiente, buscando atender as necessidades de saúde do indivíduo no seu contexto bio-psicosócio-cultural. Para assegurar a qualidade do cuidado de enfermagem nos níveis de atenção à saúde, o enfermeiro precisará ter conhecimento técnicocientífico e habilidades para atuar de forma interdisciplinar e sistematizada, no desempenho de suas atividades $^{(9)}$.

Na nova proposta curricular deverá ser incentivada a busca de informações acerca das inovações da profissão por meio da produção, utilização e disponibilização de pesquisas. Nesse contexto, o curso deverá proporcionar condições para que o futuro enfermeiro possa desenvolver suas atividades sempre de maneira crítico-reflexiva ${ }^{(9)}$.

\section{CONCLUSÃO}

A qualidade pedagógica deve fundamentar uma formação com qualificação humana, crítica e competente. Isto implica que esta formação deva ser assegurada pelo currículo, cuja construção deve ser encarada como uma construção social, visto que este é o reflexo de uma ideologia do grupo. Também envolve questões epistemológicas e a transformação das pessoas.

A inovação reside em mudar a maneira de focar o ensino direcionando as metas para a educação ao invés do treinamento, para a compreensão e não só para a técnica, levando o aluno ao desenvolvimento da consciência critica ${ }^{(10)}$.

No processo de reformulação curricular do Curso de Enfermagem da UFSCar, nem todos os envolvidos buscaram comprometer-se com o processo na mesma intensidade. Houve resistência à mudança, embora houvesse consenso que a transformação na formação passaria necessariamente pela construção de um novo projeto pedagógico, a partir das condições objetivas e subjetivas dos atores envolvidos.

As inúmeras discussões realizadas pelo grupo, por várias vezes levaram a acreditar que o processo não caminhava ou pior, que a cada encontro retrocedia. No entanto, à medida que vivenciávamos estas experiências cada vez mais nos sentíamos comprometidas com a necessidade de concluirmos o processo.

Assim sendo, conseguimos vencer o desafio com a ajuda dos demais docentes envolvidos, finalizando a primeira etapa da reformulação curricular do curso. Sua implantação está prevista para o ano de 2005 e apesar do trabalho árduo para a elaboração de um novo currículo, sabemos que foi apenas um primeiro momento de uma longa jornada. A nova etapa compreenderá o acompanhamento e análise do processo de implementação buscando superar as dificuldades de forma a assegurar uma 
Sílvia H.Zem-Mascarenhas Maria Isabel Ruiz Beretta prática pedagógica consistente com o perfil do profissional de enfermagem a ser formado na UFSCar.

Nessas idas e vindas, discussões e reflexões, o Curso de Enfermagem construiu uma proposta de currículo integrado que busca articular teoria/prática sem fragmentação, a qual representa um esforço comum dos docentes do curso.

\section{REFERÊNCIAS}

(1) Freitas MC, Guedes MVC, Silva LF. Curso de enfermagem da Universidade Estadual do Ceará - A história e projeto político-pedagógico atual. Rev Bras Enferm 2003; 56(4):385-7.

(2) Germano RM. O ensino de enfermagem em tempos de mudança. Rev Bras Enferm 2003; 56(4):365-8.

(3) Saupe R, Cestari ME. O trabalho coletivo na construção do projeto político pedagógico dos cursos de enfermagem. Rev Eletrôn Enferm [periódico online] 2002; 4(2):22-6. Disponível em: http://www.fen. ufg.br/revista> (10 mar. 2004)

(4) Mallmann MT, Daudt SID. A nova graduação da UNISINOS: compartilhando a experiência de um ato pedagógico. Rev Bras Enferm 2003; 56(4):353-7.

(5) Timoteo RPS, Liberalino FN. Reflexões acerca do fazer pedagógico a partir de referências e diretrizes educacionais para a formação em enfermagem. Rev Bras Enferm 2003; 56(4):358-60.
Entendemos que nossa trajetória, apesar de exaustiva, foi apenas o início de um caminhar e que ainda serão necessários muitos esforços por parte dos docentes, alunos e estrutura administrativa da universidade, para superar as dificuldades e garantir o sucesso de sua implementação.
(6) Feuerwerker L, Almeida M. Diretrizes curriculares e projetos pedagógicos: é tempo de ação. Rev Bras Enferm 2003; 56(4):351-2.

(7) Cunha MI. O professor universitário na transição de paradigmas. Araraquara: JM; 1998

(8) Silva RM, Gurgel AH, Moura ERF. Ética no processo ensino-aprendizagem em enfermagem obstétrica. Rev Esc Enferm USP 2004; 38(1):28-36.

(9) Universidade Federal de São Carlos. Centro de Ciências Biológicas e da Saúde. Projeto pedagógico do curso de enfermagem. São Carlos: UFSC/CCBS; 2004.

(10) Angelo M. Educação em enfermagem. Rev Esc Enferm USP 1994; 28(1):11-4.
Correspondência: Silvia H. Zem-Mascarenhas Rod. Washington Luis (SP-310), São Carlos CEP - 13565-905- SP

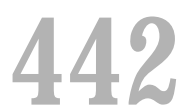

Rev Esc Enferm USP 2005; 39(4):437-42. 